\title{
Erratum: Sheathless hydrodynamic positioning of buoyant drops and bubbles inside microchannels [Phys. Rev. E 84, 036302 (2011)]
}

Claudiu A. Stan, Laura Guglielmini, Audrey K. Ellerbee, Daniel Caviezel, Howard A. Stone, and George M. Whitesides

(Received 2 February 2017; published 23 February 2017)

DOI: 10.1103/PhysRevE.95.029902

On p. 4, Eq. (10) has a misprint in the signs in a formula. The correct equation is as follows:

$$
f(\kappa)=\frac{128 \pi}{(\kappa+1)^{3}}\left(\frac{11 \kappa+10}{140}\left(3 \kappa^{2}-\kappa+8\right)-\frac{3}{14} \frac{19 \kappa+16}{3 \kappa+2}\left(2 \kappa^{2}+\kappa-1\right)\right) .
$$

This typing error does not affect the results presented in the paper. We used the correct form (shown above) of Eq. (10) to derive the data shown in the last column of Table I from Appendix A.

We would like to thank Professor R. Larson for pointing us to this error. 